\title{
Primary B-Cell Lymphoma of Caecum Presenting as an Ulcerative Lesion in a Young Male
}

\author{
Arvind Bamanikar $^{1} \quad$ Swapnil Patil ${ }^{1} \quad$ Shivam Sharma ${ }^{1}$ \\ ${ }^{1}$ Department of Medicine, Dr. D. Y. Patil Medical College, Pune, \\ Maharashtra, India

\begin{abstract}
Address for correspondence Bamanikar Arvind, FRCP, Department of Medicine, Dr. D. Y. Patil Medical College, Dr. DY Patil Vidyapeeth, Sant Tukaram Nagar, Pune 411018, Maharashtra, India (e-mail: arvind045@gmail.com).
\end{abstract}

\begin{abstract}
Keywords

- non-Hodgkin's

lymphoma

- extra nodal

- cecum

$-B C L U$

Extra nodal site of non-Hodgkin's lymphoma is mostly in the gastrointestinal tract. Large intestine is rarely affected compared with stomach or small bowel. The colon is affected in $0.4 \%$ of cases approximately. High index of suspicion is required to establish the diagnosis, since there may be no specific signs. Here we report a case of a 28-year-old male, who presented with diarrhea , abdominal pain and weight loss of 2-month duration and diagnosed as non-Hodgkin's lymphoma (NHL) of the cecum as B-cell lymphoma, unclassified (BCLU) according to World Health Organization (WHO) guidelines.
\end{abstract}

\section{Introduction}

Non-Hodgkin's lymphomas (NHLs) commonly present as lymphadenopathy. About $85 \%$ of all malignant lymphomas are NHLs. Burkitt's lymphoma and lymphoblastic lymphoma occurs in younger patients. One-third of NHLs involve the extra nodal sites. ${ }^{1}$ Primary NHL accounts for 0.1 to $0.5 \%$ of all colorectal malignant disease and the most common histological subtype occurring in the colorectal lymphoma is diffuse large B-cell type with 47 to $81 \%$ of occurrence. ${ }^{2}$

\section{Case History}

A 28-year-old male presented with diarrhea and weight loss of $5 \mathrm{~kg}$ over a period of 6 months and frequent episodes of abdominal pain since 2 months. Clinical examination was normal. Routine hematology and biochemistry was normal. Serology for HBV and HIV was negative.

Colonoscopy showed small discrete ulcers measuring less than $1 \mathrm{~cm}$ in the cecum ( $\mathbf{- F i g . ~ 1 ) ~ w i t h ~ m u c o s a l ~ t h i c k e n i n g ~ a t ~}$ the distal portion of the ascending colon. Contrast-enhanced computed tomography (CECT) abdomen showed asymmetrical thickening (maximum $11 \mathrm{~mm}$ ) of the cecal wall with moderate thickening of ileocecal valve. Other organs were normal.

Microscopy with routine showed ulcerated cecal mucosa with sub mucosa and muscularis propria showing diffuse infiltration of monomorphic neoplastic lymphoid cells which were large, round with vesicular nuclei, and prominent nucleoli (-Figs. 2-4). Immunohistochemistry with specific markers, CD20/CD3/CD5 showed positive results and CD10/ $\mathrm{TdT} /$ cyclineD1 having negative results confirmed the diagnosis of B-cell lymphoma. (NHL).The diagnosis according to World Health Organization (WHO) classification was B-cell lymphoma, unclassified. (BCLU)

CD20 is the most widely used pan-B-cell marker and is expressed from the narve $\mathrm{B}$-cell until the final stages of B-cell development just prior to plasmacytic differentiation.

Positron emission tomography (PET) scan performed with $7.5 \mathrm{mCi}$ of F-18 2-deoxyglucose (FDG) showed

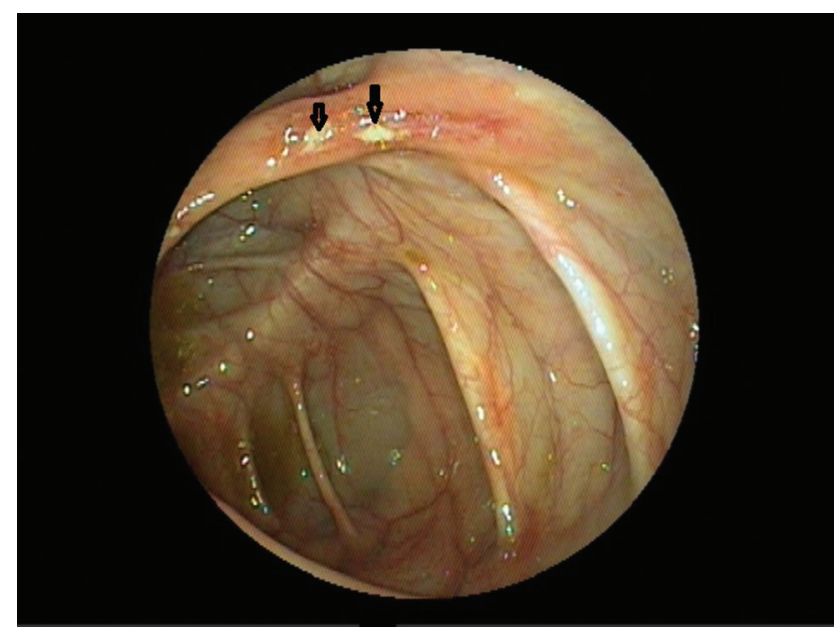

Fig. 1 Ulcers seen (arrows) in the cecum on colonoscopy. 


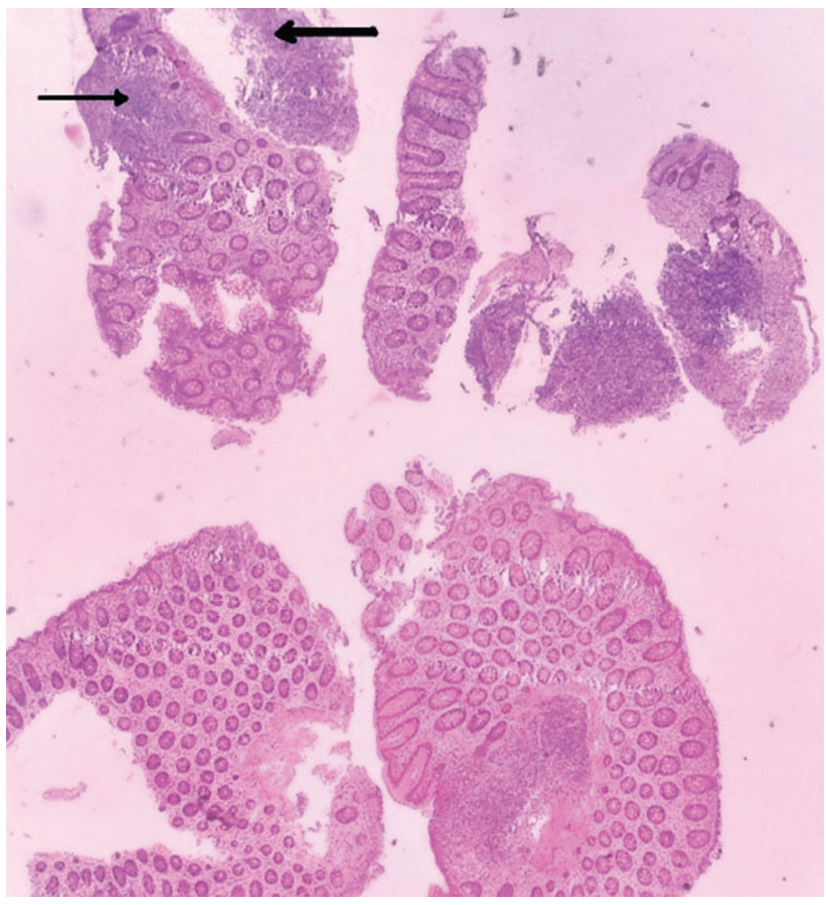

Fig. 2 Photomicrograph of colonic biopsy fragments showing nodular lymphoma aggregates in mucosa (thin arrow) and beneath the muscularis mucosa (thick arrow).

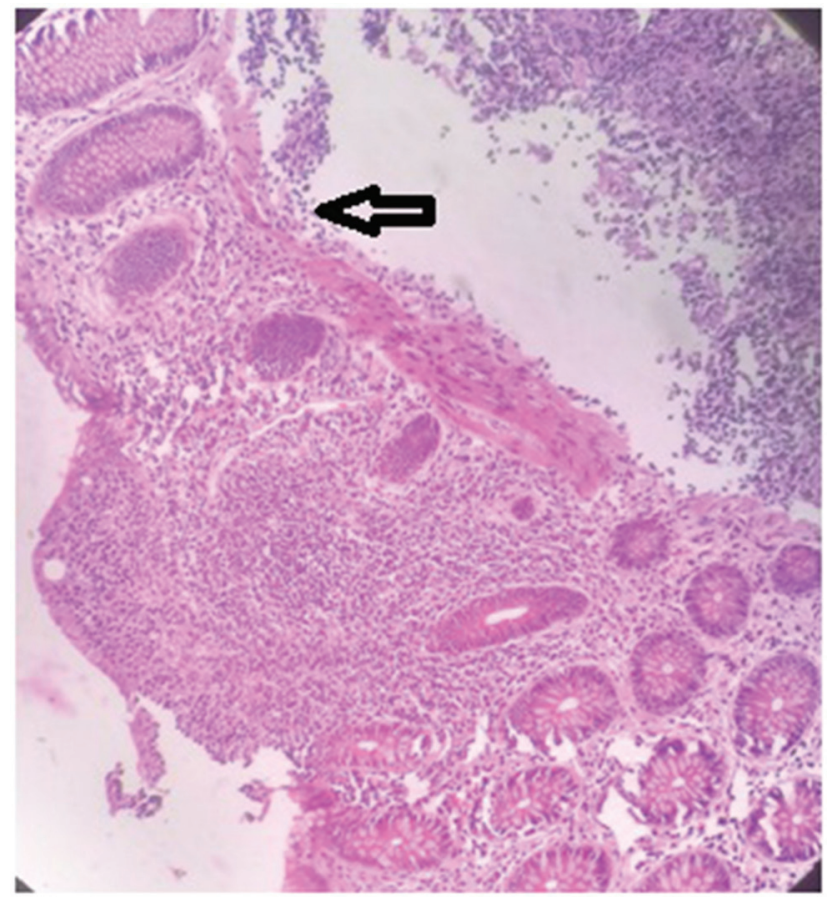

Fig. 3 Photomicrograph showing extensive neoplastic lymphoid infiltrate in the mucosa and disrupting the muscularis mucosa (arrow).

circumferential thickening of cecum. FDG uptake was seen in multiple mesenteric nodes measuring $1.5 \times 1.2 \mathrm{~cm}$, subcentric aortocaval node, mildly enhancing mesenteric thickening along the SMV (superior mesenteric vein). The conclusion was the patient has metabolically active cecal wall thickening consistent with histology diagnosis of

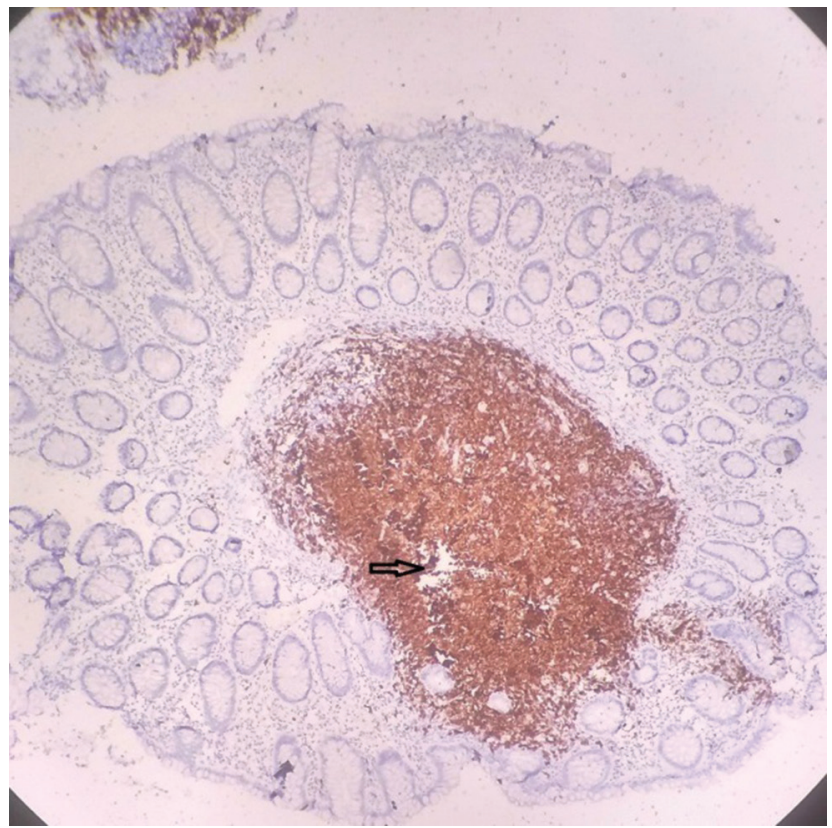

Fig. 4 Photomicrograph showing lymphoid aggregates in the mucosa and submucosa show a striking predominance of CD20 positive B-cells admixed with scattered $C D 3$ and $C D 5$ positive T-cells. The aggregates are negative for CD10, TdT, terminal deoxynucleotidyl transferase, and Cyclin D1.

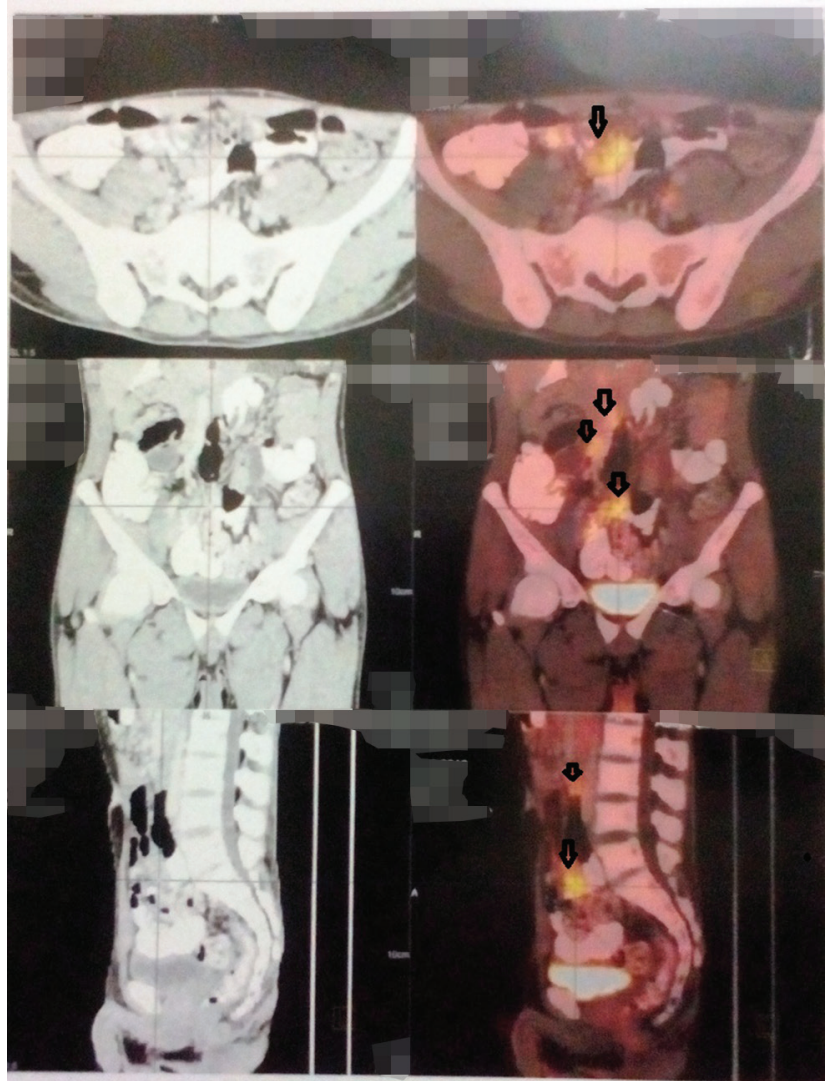

Fig. 5 PET scan showing metabolically active lesions (cecum and nodes). PET, positron emission tomography.

lymphoma. The presence of metabolically active mesenteric and aortocaval, cervical nodes were noted ( - Fig. 5 ). 
The staging of the index case in our report of BCLU was T3N3M0B0 according to Paris Staging system which is specifically meant for primary gastrointestinal (GI) lymphomas.

\section{Discussion}

NHLs are tumors originating from lymphoid tissues, mainly of lymph nodes. About $85 \%$ of all malignant lymphomas are NHLs. These tumors may result from chromosomal translocations, infections, environmental factors, immunodeficiency states, and chronic inflammation. ${ }^{2}$ Colon is an uncommon site of involvement in NHL. The most common symptoms of colonic lymphoma are abdominal pain and weight loss, with a palpable abdominal mass. ${ }^{3}$ The standard criteria for the diagnosis of primary intestinal lymphoma were established in 1961 by Dawson et al. ${ }^{4}$

In the Ann Arbor classification, no distinction is made between primary nodal and primary extra nodal lymphomas, clearly because primary extra nodal involvement is extremely rare in patients with Hodgkin's disease, for whom this classification was originally designed. Strict criteria were proposed in 1961 by Dawson et al in a study on patients included only if superficial lymph nodes were not palpable at first examination, absence of significant enlargement of mediastinal lymph nodes on chest X-ray, and the normal white blood cell count. Furthermore, upon laparotomy, the bowel lesion had to be shown to be dominant and only involvement of lymph nodes in the immediate vicinity of the primary lesion was acceptable. The National Cancer Database report on NHL (1997) from hospitals in the United States used the site of tumor as the only criterion for primary nodal or extra nodal disease. ${ }^{5}$

Tumors were considered to be primary if (1) no palpable superficial lymphadenopathy at the first examination; (2) no obvious enlargement of the mediastinal nodes on chest X-ray; (3) normal white blood cell counts, total and differential, normal bone marrow; (4) no focal lesions in the liver, spleen, and bone on single-photon emission computed tomography (SPECT) scan and only lymph nodes obviously affected in the immediate neighborhood; and (5) no FDG uptake in liver and spleen.

Krol et $\mathrm{al}^{5}$ described three alternative definitions of primary nodal and extra nodal NHL, exploring their effect on the percentage of the patients considered to have primary extra nodal disease, their treatment outcome and survival in a population-based cohort of NHL patients.

In the modern era, these criteria have been expanded to the newer diagnostic tools.

The most common histological subtype occurring in the colon and rectum is diffuse large B-cell lymphoma with frequency ranging from 47 to $81 \%$ depending on the geographical location. ${ }^{6,7}$

Most of T-cell lymphomas of the gut present usually as ulcers or strictures in the endoscopic examinations, while primary B-cell lymphomas commonly present as exophytic lesions. ${ }^{8}$

\section{Staging of Lymphoma}

\section{Histology}

The Ann Arbor staging system is suboptimal for documentation of the specific features of primary lymphoma in the GI tract. Several modifications and alternatives have been proposed like the modified An Arbor, Lugano, and Paris staging. Tumor nodes metastasis (TNM) staging for tumors of epithelial origin has also been proposed as an alternative in GI lymphoma to describe localized disease.

Primary intestinal lymphoma is mostly seen in the ileocecal region, probably due to presence of high lymphoid tissue. Involvement of regional lymph nodes has a bad prognosis ${ }^{2,9}$ Clinical diagnosis of GI tract lymphoma is very difficult and often needs surgical specimen for histological diagnosis of NHL; B-cell or T-cell type. B-cell type has better prognosis as compared with T-cell lymphoma. ${ }^{10}$ Immunohistochemistry is a useful adjunct in subclassification of NHL pan-B-cell markers such as CD20, CD19, CD22, CD45, and CD79a are generally expressed in BCLU. CD10 is expressed in 30 to $60 \%$ of cases. CD10 is a marker for follicular center B-cell differentiation. Seki et al reported that the presence of CD10 expression was associated with improved survival in patients with BCLU; hence, CD10 expression may be useful for determining the prognosis of BCLU.11

\section{PET for Risk Stratification of Pretreatment}

PET-CT was recommended in updated international guidelines for staging/restaging of B-cell lymphoma (BCLU) and follicular lymphoma (FL).

Whole body FDG PET scan has a role in the initial evaluation of patients with NHL; however, it is of more value in posttreatment scenario to differentiate between early recurrences or residual disease and fibrosis or necrosis. According to current data, BCLU intermediate between diffuse large B-cell lymphoma and Burkitt's lymphoma represents a highly aggressive type of lymphoma with dramatically bad response for chemotherapy. Our patient had B-lymphoma spread beyond the regional nodes and required chemotherapy.

\section{Conclusion}

The diagnosis of primary colonic lymphoma is rarely made on colonoscopy as a first line investigation but certainly provides tissue from the lesions for confirmation by histopathological methods. The histological type is important, since BCLU is curable with surgery and or chemotherapy. Thus lymphoma should be considered as a differential diagnosis in patients presenting with colonic ulcers.

\section{References}

1 Kumar V, Abbas AK, Aster JC. Diseases of white blood cells, lymph nodes, spleen and thymus. Robbins and Cotran Pathologic Basis of Disease. Vol. 1, 9th ed. India: Reed Elsevier; 2014 579-628

2 Kumar KM, Indira V, Reddy CK, Swetha K, Vujhini SK. Diffuse large B-cell lymphoma of the caecum in a non-HIV patient - a rare occurrence: case report. Sch Acad J Biosci 2014;2(9):613-617 
3 Bairey O, Ruchlemer R, Shpilberg O. Non-Hodgkin's lymphomas of the colon. Isr Med Assoc J 2006;8(12):832-835

4 Dawson IM, Cornes JS, Morson BC. Primary malignant lymphoid tumours of the intestinal tract. Report of 37 cases with a study of factors influencing prognosis. Br J Surg 1961;49:80-89

5 Krol AD, le Cessie S, Snijder S, Kluin-Nelemans JC, Kluin PM, Noordijk EM. Primary extranodal non-Hodgkin's lymphoma (NHL): the impact of alternative definitions tested in the comprehensive cancer centre West population-based NHL registry. Ann Oncol 2003;14(1):131-139

6 Cai S, Cannizzo F Jr, Bullard Dunn KM, Gibbs JF, Czuczman $\mathrm{M}$, Rajput A. The role of surgical intervention in non-Hodgkin's lymphoma of the colon and rectum. Am J Surg 2007;193(3):409-412, discussion 412

7 Gavriilidis P, Christoforidou B, Michalopoulou I, Nikolaidou A. Primary ileocaecal B-cell non-Hodgkin lymphoma (NHL): a rare underlying cause of right iliac fossa pain. BMJ Case Rep 2013;2013:bcr2013009052

8 Wu PH, Chu KE, Lin YM, Huang SH, Wu CC. T-Cell Lymphomas Presenting as Colon Ulcers and Eosinophilia. Case Rep Gastroenterol 2015;9(2):246-252

9 Jayabackthan L, Murgi SB, Graham S, Kini RG. A rare case of primary lymphoma of the caecum presenting as intussusception. J Lab Physicians 2013;5(2):118-120

10 Risio D, Percario R, Legnini M, et al. Diffuse large B-cell lymphoma of the colon with synchronous liver metastasis: a rare case report mimicking metastatic colorectal adenocarcinoma. BMC Surg 2014;14:75

11 Seki R, Ohshima K, Fujisak T, et al. Prognostic impact of immunohistochemical biomarkers in diffuse large B-cell lymphoma in the rituximab era, Cancer Sci, 2019;100(10):1842-1847 